Wright State University

CORE Scholar

Physics Faculty Publications

Physics

7-1-2007

\title{
Role of Subsurface Defects in Metal-ZnO(0001) Schottky Barrier Formation
}

\author{
H. L. Mosbacker \\ S. El Hage \\ M. Gonzalez \\ S. A. Ringel \\ M. J. Hetzer
}

See next page for additional authors

Follow this and additional works at: https://corescholar.libraries.wright.edu/physics

Part of the Physics Commons

\section{Repository Citation}

Mosbacker, H. L., El Hage, S., Gonzalez, M., Ringel, S. A., Hetzer, M. J., Look, D. C., Cantwell, G., Zhang, J., Song, J. J., \& Brillson, L. J. (2007). Role of Subsurface Defects in Metal-ZnO(0001) Schottky Barrier Formation. Journal of Vacuum Science \& Technology B, 25 (4), 1405-1411.

https://corescholar.libraries.wright.edu/physics/3

This Article is brought to you for free and open access by the Physics at CORE Scholar. It has been accepted for inclusion in Physics Faculty Publications by an authorized administrator of CORE Scholar. For more information, please contact library-corescholar@wright.edu. 


\section{Authors}

H. L. Mosbacker, S. El Hage, M. Gonzalez, S. A. Ringel, M. J. Hetzer, David C. Look, G. Cantwell, J. Zhang, J. J. Song, and L. J. Brillson

This article is available at CORE Scholar: https://corescholar.libraries.wright.edu/physics/3 


\title{
Role of subsurface defects in metal-ZnO(0001) Schottky barrier formation
}

\author{
H. L. Mosbacker, ${ }^{\text {a),b) }}$ S. El Hage, M. Gonzalez, and S. A. Ringel ${ }^{\text {b) }}$ \\ Department of Electrical and Computer Engineering, The Ohio State University, \\ Columbus, Ohio 43210 \\ M. Hetzer \\ Department of Physics, The Ohio State University, Columbus, Ohio 43210 \\ D. C. Look $^{\text {() }}$ \\ Air Force Research Laboratory, AFRL/MLPS, Wright-Patterson Air Force Base, Ohio 45435 \\ G. Cantwell, J. Zhang, and J. J. Song ${ }^{\text {d) }}$ \\ ZN Technology, Inc., 910 Columbia St., Brea, California 92821 \\ L. J. Brillson ${ }^{\text {b),e) }}$ \\ Department of Electrical and Computer Engineering, The Ohio State University, \\ Columbus, Ohio 43210
}

(Received 14 December 2006; accepted 11 June 2007; published 30 July 2007)

\begin{abstract}
The authors fabricated diodes of $\mathrm{Au}, \mathrm{Al}, \mathrm{Ni}, \mathrm{Pt}, \mathrm{Pd}, \mathrm{Mo}$, Ta, and Ir on single crystal $\mathrm{ZnO}(000 \overline{1})$ surfaces from different vendors and measured their Schottky barriers, idealities, and reverse currents on as-received and remote oxygen $\left(20 \% \mathrm{O}_{2} / 80 \% \mathrm{He}\right)$ plasma-treated surfaces. Using low temperature nanoscale depth-resolved cathodoluminescence spectroscopy (DRCLS) under the metal, the authors identified the presence of defect transitions at 2.1,2.5, and $3.0 \mathrm{eV}$ that change dramatically depending on the process steps and choice of metal. $I-V$ measurements exhibited transitions from Ohmic to Schottky and lower idealities for Pt, Au, Ir, and Pd with plasma treatment. $\mathrm{ZnO}$ with low defect densities yield lower idealities and reverse currents. Deep level optical and transient spectroscopies correlated bulk and surface defects, showing deep levels at 2.54 and $0.53 \mathrm{eV}$, while DRCLS shows that these densities can increase by $>100$ times at the surface. The magnitude of the metal's influence correlates directly to the relative defect concentrations near the surface and in the bulk. These results show that metals can induce defect states at the metal-semiconductor interface and impact device performance. () 2007 American Vacuum Society. [DOI: 10.1116/1.2756543]
\end{abstract}

\section{INTRODUCTION}

$\mathrm{ZnO}$ has emerged as an important material for its superior electronic and optoelectronic properties with applications that include UV and blue light emission, room temperature spintronic devices, and novel nanostructures for electronics and materials. ${ }^{1-4}$ Realization of such device applications will require a detailed understanding of clean, ordered metal/ $\mathrm{ZnO}$ interfaces. However, multiple-metal studies on clean $\mathrm{ZnO}$ surfaces from multiple sources have until now not been conducted. Previous work has largely been phenomenological in nature or has focused on the presence of interfacial contamination. ${ }^{5-11}$ Few studies have tried to understand the role of subsurface or bulk states in the semiconductor, or the effect of mobile contaminants such as Li or $\mathrm{H}^{12-14}$ Through the use of in situ plasma cleaning and metal deposition, we

a)Electronic mail: mosbacker.1@osu.edu

${ }^{b}$ Also at Department of Physics, The Ohio State University, Columbus, $\mathrm{OH}$ 43210.

${ }^{c}$ Also at Semiconductor Research Center, Wright State University, Dayton, OH 45435.

d) Also at Department of Electrical and Computer Engineering, University of California, San Diego, La Jolla, CA 92093.

e) Also at Center for Materials Research, The Ohio State University, Columbus, $\mathrm{OH} 43210$. have been able to show that surface contamination, bulk states, and states near the surface, as well as hydrogen, affect electrical contacts in $\mathrm{ZnO}^{12-14}$ In this article, we extend these themes and measure the effect of each contribution on Schottky barrier (SB) formation at the metal- $\mathrm{ZnO}$ interface. We deposited eight metals ( $\mathrm{Au}, \mathrm{Al}, \mathrm{Ir}, \mathrm{Ni}, \mathrm{Mo}, \mathrm{Pd}, \mathrm{Pt}$, and Ta) on untreated and $\mathrm{O}_{2}$ plasma-treated surfaces from different vendors and correlated electrical measurements systematically with (i) plasma processing, (ii) the presence of states in the subsurface and bulk, and (iii) defects induced at metal/ $\mathrm{ZnO}$ interfaces. These results demonstrate that subsurface and metal interface defects in $\mathrm{ZnO}$ single crystals have a major effect on Schottky barrier characteristics.

\section{EXPERIMENTAL PROCEDURE}

We examined both melt-grown and hydrothermally grown single crystal substrates of $\mathrm{ZnO}$. The surfaces were chemomechanically polished by their manufacturer. All substrates were subjected to a standard rinse for $5 \mathrm{~min}$ each in acetone, toluene, dimethyl-sulfoxide, methanol, and de-ionized water, after which the samples were blown dry in nitrogen. One set of samples from each vendor were subjected to a $20 \%$ $\mathrm{O}_{2} / 80 \% \mathrm{He}$ inductively coupled remote oxygen plasma (ROP) with a background pressure of $10 \mathrm{mTorr}$. Gas concen- 


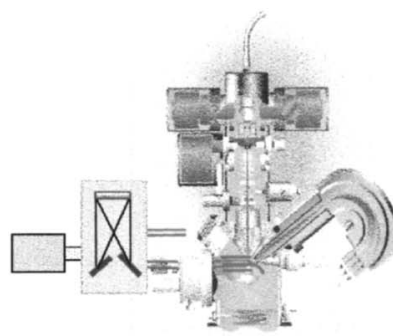

(a)

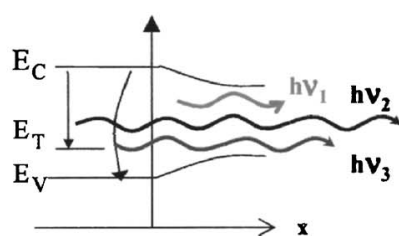

(b)

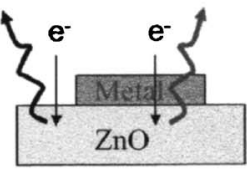

(c)
FIG. 1. (a) JEOL 7800F UHV SEM chamber with adjoining monochromator and detectors for DRCLS. (b) Optical transitions excited by DRCLS including band to band, band to defect, and new interface compound transitions. (c) Incident electrons and exciting photons near and through $30 \mathrm{~nm}$ $\mathrm{Al}$ metal diode on $\mathrm{ZnO}$. trations were maintained with mass flow controllers, and were mixed before entering the process chamber. The rf plasma power was maintained at a constant $33 \mathrm{~W}$. The ROPtreated samples were processed for $1 \mathrm{~h}$; then $0.4 \mathrm{~mm}$ diameter circular contacts were electron beam deposited through a shadow mask to a thickness of $30 \mathrm{~nm}$. These contacts are thin enough to permit detection of luminescence through the diodes. We produced both in situ and ex situ contacts for each vendor's $\mathrm{ZnO}$ and each surface process. X-ray photoelectron spectroscopy (XPS) and low energy electron diffraction were previously used to verify clean ordered surfaces for metal deposition. ${ }^{14}$ Atomic force microscopy (AFM) shows that the ROP process does not damage the surface of the material, ${ }^{14}$ and depth-resolved cathodoluminescence spectroscopy (DRCLS) indicates that the process reduces defectrelated luminescence in the near surface region. ${ }^{14}$ AFM shows a mild increase of rms surface roughness from 0.1 to $0.2 \mathrm{~nm}$ while DRCLS reveals the reduction of point defect luminescence by a factor of 5.0. ${ }^{14,15}$

We performed current-voltage $(I-V)$ measurements following DRCLS studies using a HP 4145B semiconductor analyzer. Capacitance-voltage $(C-V)$, deep level transient spectroscopy (DLTS) measurements, and deep level optical spectroscopy (DLOS), as described elsewhere, ${ }^{16}$ were performed on Schottky diodes.

DRCLS spectra were taken using a JEOL 7800F ultrahigh vacuum (UHV) scanning electron microscope (SEM) [Fig. 1(a)]. A parabolic mirror connected to a photomultiplier collected the luminescence. The sample was cooled with an Oxford stage using liquid He to a temperature of $10 \mathrm{~K}$. An Oxford MonoCL monochromator was used to record the spectra with a maximum resolution of $0.15 \mathrm{~nm}$. The SEM electron beam was varied with constant power from 2 to $20 \mathrm{keV}$ with a maximum spot size of $50 \mathrm{~nm}$. DRCLSs were acquired though the metal diode and on the free surface, as shown in Fig. 1(b). Transmission measurements were carried out that indicated negligible light absorption in the $1.8-3.0 \mathrm{eV}$ region of interest. Monte Carlo simulations ${ }^{17}$ of electron energy loss and electron-hole $(e-h)$ pair creation were calculated to determine the electron beam penetration through the metal and into the bulk crystal. For electron beam energies $E_{B}=2,5,10$, and $20 \mathrm{keV}$, depths of peak $e-h$ pair excitation rate $U_{0}$ were $55,85,330$, and $990 \mathrm{~nm}$, respectively. In general, these results indicated that both a $2 \mathrm{keV}$ beam at the free surface and a $5 \mathrm{keV}$ beam through the $30 \mathrm{~nm}$ thick metals penetrate $\sim 40 \mathrm{~nm}$ into the $\mathrm{ZnO}$. All measurements were performed on arrays of eight diodes for each metal, all on the same $\mathrm{ZnO}$-processed surface.

\section{RESULTS}

DRCLS measurements of $\mathrm{ZnO}$ revealed pronounced deep level emissions that varied strongly with growth method, surface preparation, and subsequent metallization. A DRCLS survey of $\mathrm{ZnO}$ single crystals grown by hydrothermal, melt grown, chemical vapor transport, and molecular beam epitaxy showed orders of magnitude difference in crystal quality in terms of the magnitude of near band edge (NBE) and deep level (DL) emissions as well as their uniformity with depth. Pronounced differences are observed even within growths from the same vendor. ${ }^{18}$ This article will address effects of bulk and metal-induced defects for representative "high defect" and "low defect" ZnO materials. Thus, Fig. 2 illustrates DRCLS spectra from a high defect (a) versus a low defect
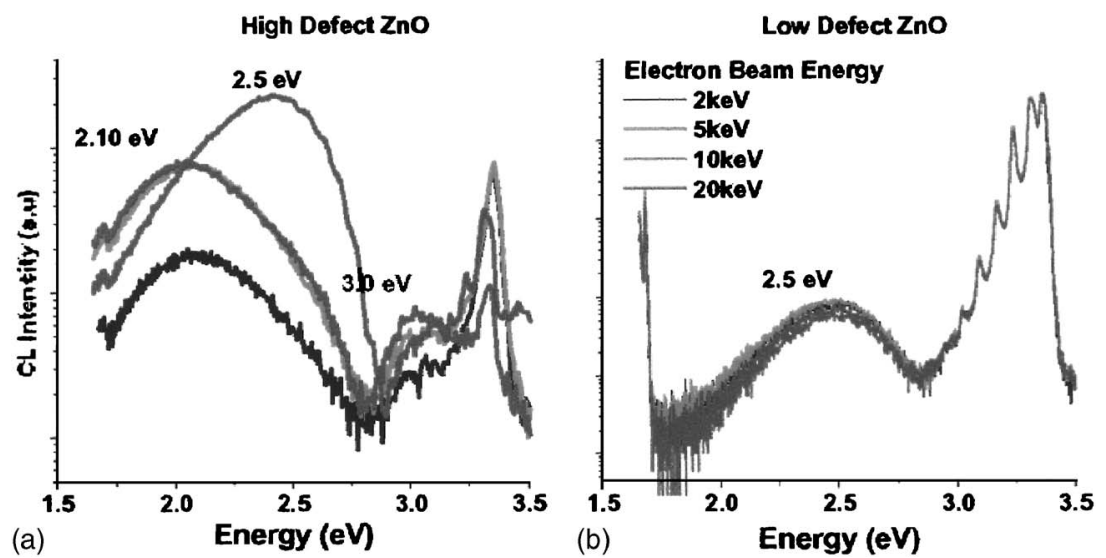

FIG. 2. Comparison of DRCLS spectra vs excitation depth for relatively (a) high and (b) low deep level defect $\mathrm{ZnO}(000 \overline{1})$ bare surfaces. High defect $\mathrm{ZnO}$ exhibits subsurface defect emissions at 2.1, 2.5, and $3 \mathrm{eV}$ that exceed NBE emission and vary by orders of magnitude vs depth over a $10-400 \mathrm{~nm}$ range. Low defect $\mathrm{ZnO}$ exhibits orders of magnitude lower deep level emission and greater uniformity over the same depth range. 


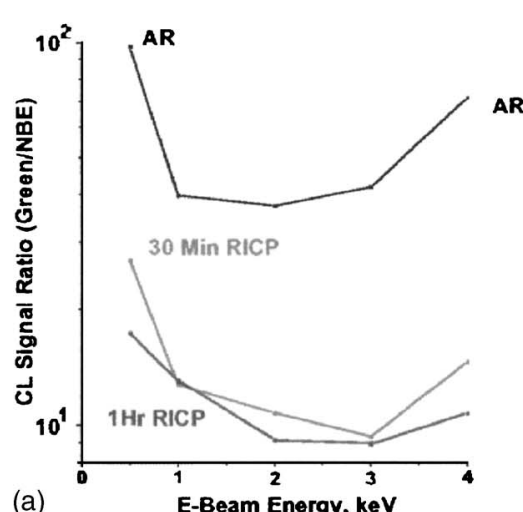

(a)

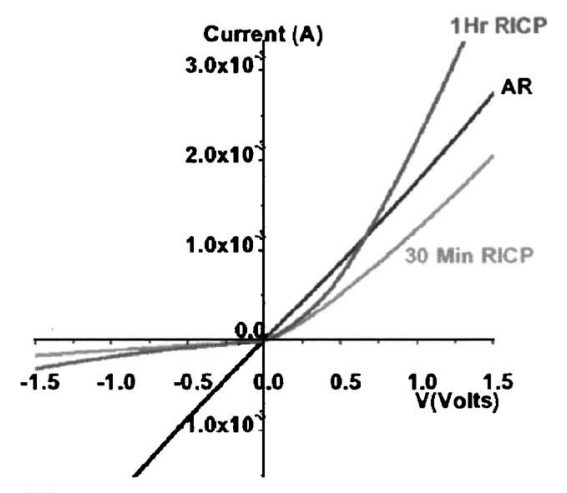

(b)
FIG. 3. (a) $I(2.5 \mathrm{eV}) / I(\mathrm{NBE})$ vs $E_{B}$ for as received and 30 and $60 \mathrm{~min}$ ROP exposures showing increase near surface and overall decrease vs ROP exposure time. (b) Corresponding $I-V$ curves showing transition from Ohmic to Schottky behavior on high defect substrates with increasing ROP treatment time. (b) $\mathrm{ZnO}(000 \overline{1})$ surface. The high defect $\mathrm{ZnO}$ exhibits broad DL emissions at approximately 2.1, 2.5, and $3.0 \mathrm{eV}$ in addition to some excitonic features in the NBE region. These DL emissions are comparable to or higher than NBE intensities and vary by orders of magnitude with $E_{B}$ and excitation depth. In contrast, the low defect $\mathrm{ZnO}$ exhibits many orders of magnitude higher NBE/DL intensity ratio and much more uniformity versus depth. The distinct series of LO phonon replicas in Fig. 2(b) are an additional indication of high crystal quality.

Previously, we showed that ROP treatment of $\mathrm{ZnO}$ surfaces removes surface adsorbates, removes hydrogen, and reduces deep level emissions at depths tens to hundreds of nanometers below the surface. ${ }^{14}$ Figure 3 illustrates the effects of ROP treatment on DL intensities versus depth and $I-V$ curves. In Fig. 3(a), the ratio of $2.5 \mathrm{eV}$ "green" DL versus NBE intensities $I(2.5 \mathrm{eV}) / I(\mathrm{NBE})$ decreases with increasing $E_{B}$ and excitation depth. After a $30 \mathrm{~min}$ ROP exposure, these ratios decrease by a factor of 3 and all surface carbon and hydroxide are removed. After a 60 min ROP exposure, $I(2.5 \mathrm{eV}) / I(\mathrm{NBE})$ decreases even further. As shown in Fig. 3(a), we consistently observe that the DL/NBE intensity beyond the first few hundred nanometers increases into the bulk for all $\mathrm{ZnO}$ substrates regardless of vendor. Furthermore, a similar depth variation appears for DRCL spectra obtained in cross section. Figure 3(b) shows the corresponding $I-V$ curves. The as-received (AR) surface exhibits a straight line, Ohmic behavior. With a $30 \mathrm{~min}$ ROP exposure, a SB with $\Phi_{B}=0.4 \mathrm{eV}$ and ideality factor $n>5$ is obtained. With 60 min ROP exposure, $\Phi_{B}$ increases to $0.5 \mathrm{eV}$ and $n$ decreases to 2.0. Thus defect removal improves SB characteristics substantially even after surface contamination is removed. Note that ideality factors extracted from $I-V$ measurements with $n>2$ are not considered reliable. They are, however, consistent with high levels of recombination and/or tunneling. More importantly, they demonstrate that ROP processing reduces these effects.

The concentration and distribution of defects below the $\mathrm{ZnO}(000 \overline{1})$ surface vary dramatically between vendors. Figure 4(a) illustrates $I(2.5 \mathrm{eV}) / I(\mathrm{NBE})$ versus depth for specimens grown from the melt (a) versus vapor phase transport (ZN Tech) (b). Here, the $I(2.5 \mathrm{eV}) / I(\mathrm{NBE})$ ratios vary by orders of magnitude and are labeled "high," "medium," and "low" defect accordingly. Figure 4(b) illustrates log $I-V$ characteristics for $\mathrm{Au}$ diodes deposited on these specimens subsequent to ROP treatment. High defect diodes exhibited high reverse currents and a $\Phi_{B}=0.43 \mathrm{eV}$. Medium defect diodes showed similar reverse currents and a slightly higher $\Phi_{B}$ $=0.5 \mathrm{eV}$. Low defect $\mathrm{ZnO}$ diodes displayed reverse currents two orders of magnitude lower and the highest $\mathrm{SB}, \Phi_{B}$ $=0.60 \mathrm{eV}$. In general, higher defect densities result in higher reverse leakage currents and lower barriers for the same metal diode.

The ROP treatment affects not only the $\mathrm{ZnO}(000 \overline{1})$ near surface chemistry and defect concentrations but also the types and intensities of defects under metal contacts. Figure 5 illustrates DRCLS spectra for $\mathrm{Au}, \mathrm{Pt}, \mathrm{Pd}, \mathrm{Ir}, \mathrm{Al}, \mathrm{Mo}, \mathrm{Ni}$, and Ta on both AR (a), i.e., unprocessed, versus ROP-treated (b) surfaces. Without processing, all metals appear to yield
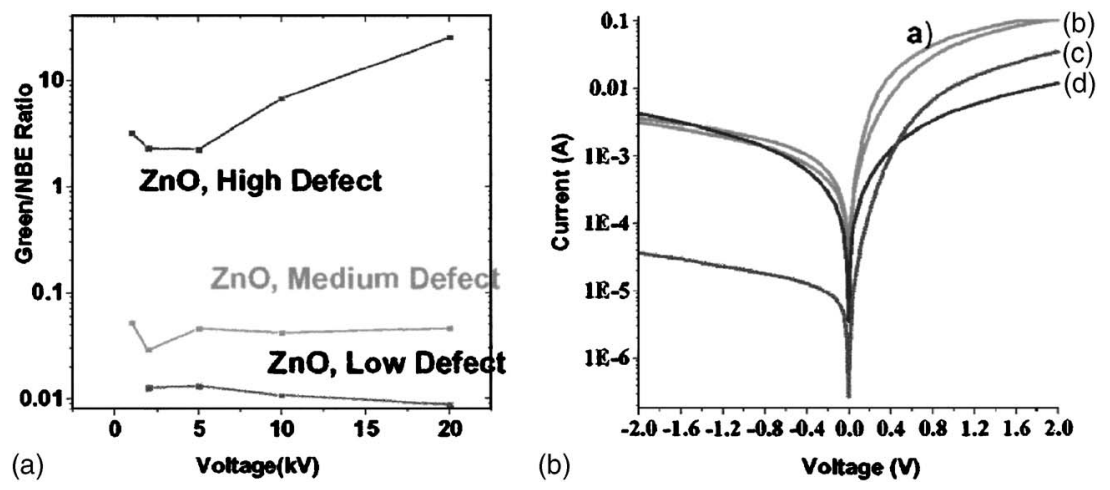

FIG. 4. (a) High, medium, and low $\mathrm{ZnO} I(2.5 \mathrm{eV}) / I(\mathrm{NBE})$ versus $E_{B}$. (b) $\log I-V$ curves for the corresponding Au diodes of [(a) and (b)] medium (c) low, and (d) high defect $\mathrm{ZnO}$. Reverse leakage current increases and SB decreases with increasing defect ratio.

JVST B - Microelectronics and Nanometer Structures 

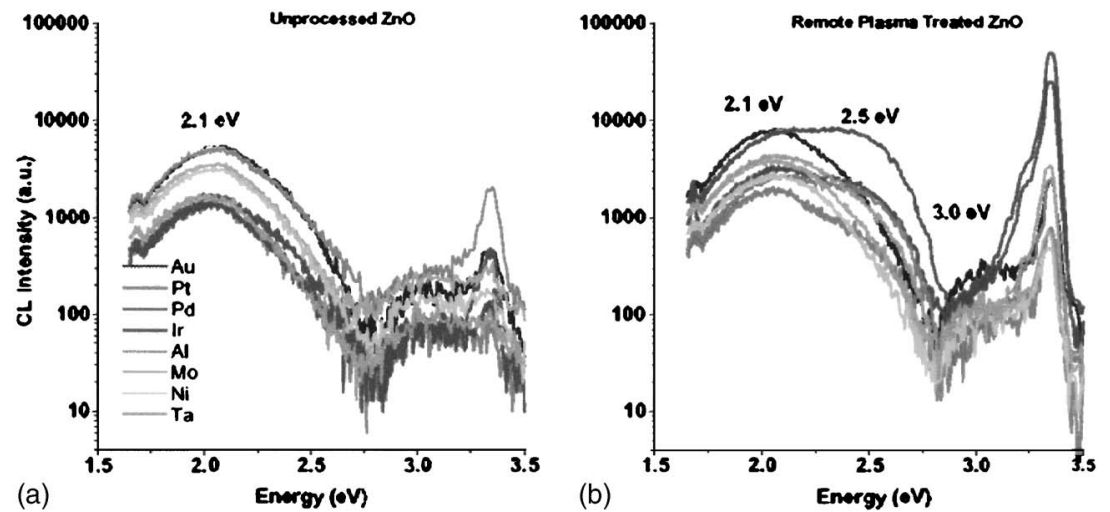

FIG. 5. DRCL spectra with $E_{B}$ $=5 \mathrm{keV}$ obtained through diodes of the metals indicated, all on the same (a) AR high defect material vs (b) ROP-treated $\mathrm{ZnO}(000 \overline{1})$ surface. The AR spectra are relatively independent of metal, in contrast to the ROPtreated surface spectra.

qualitatively similar spectra with defect emission at $2.1 \mathrm{eV}$ exceeding NBE intensities by more than an order of magnitude. Significant deep level emission is also evident at $\sim 3 \mathrm{eV}$ in all cases. In contrast, ROP-treated $\mathrm{ZnO}(000 \overline{1})$ spectra exhibit orders of magnitude greater NBE emission and substantial differences between metals. In particular, 2.1, 2.5 , and $3 \mathrm{eV}$ emissions appear to vary significantly depending on the metal, both in relative intensity and, in the case of the $\sim 3 \mathrm{eV}$ band, linewidth as well.

The ROP treatment also has a dramatic effect on the Schottky barriers for these metals. Figure 6 shows $\log I-V$ characteristics for $\mathrm{Au}, \mathrm{Pd}, \mathrm{Pt}$, and Ir for AR versus ROPtreated surface diodes with the same high defect $\mathrm{ZnO}$ crystal. As already presented, the $\mathrm{Au}$ diodes were Ohmic prior to plasma treatment but had $\Phi_{B}=0.43 \mathrm{eV}$ and $n=3.7$ for the plasma-treated surface. Similarly, Pt diodes were Ohmic for AR surfaces but showed $\Phi_{B}=0.42 \mathrm{eV}$ and $n=3.45$ for the ROP-treated surface. Pd diodes had $\Phi_{B}=0.49 \mathrm{eV}$ and $n$ $=1.48$ before ROP treatment and $\Phi_{B}=0.70 \mathrm{eV}$ and $n=1.5$ after ROP processing. Finally, the AR diodes for Ir showed $\Phi_{B}=0.65 \mathrm{eV}$ and $n=2.42(\mathrm{AR})$ versus $\Phi_{B}=0.69 \mathrm{eV}$ and $n$ $=1.58$ post-ROP. In each case, $\Phi_{B}$ increases and $n$ decreases with ROP cleaning and defect reduction. The corresponding $\Phi_{B}$ 's and ideality factors for low defect. AR versus ROPtreated material are summarized in Table I. Similarly, $\Phi_{B}$ 's and ideality factors for low versus high defect materials are summarized in Table I.

DLTS, DLOS, as well as capacitance-voltage $(C-V)$ measurements were performed on the high defect material in order to obtain DL concentrations, their gap state energies,
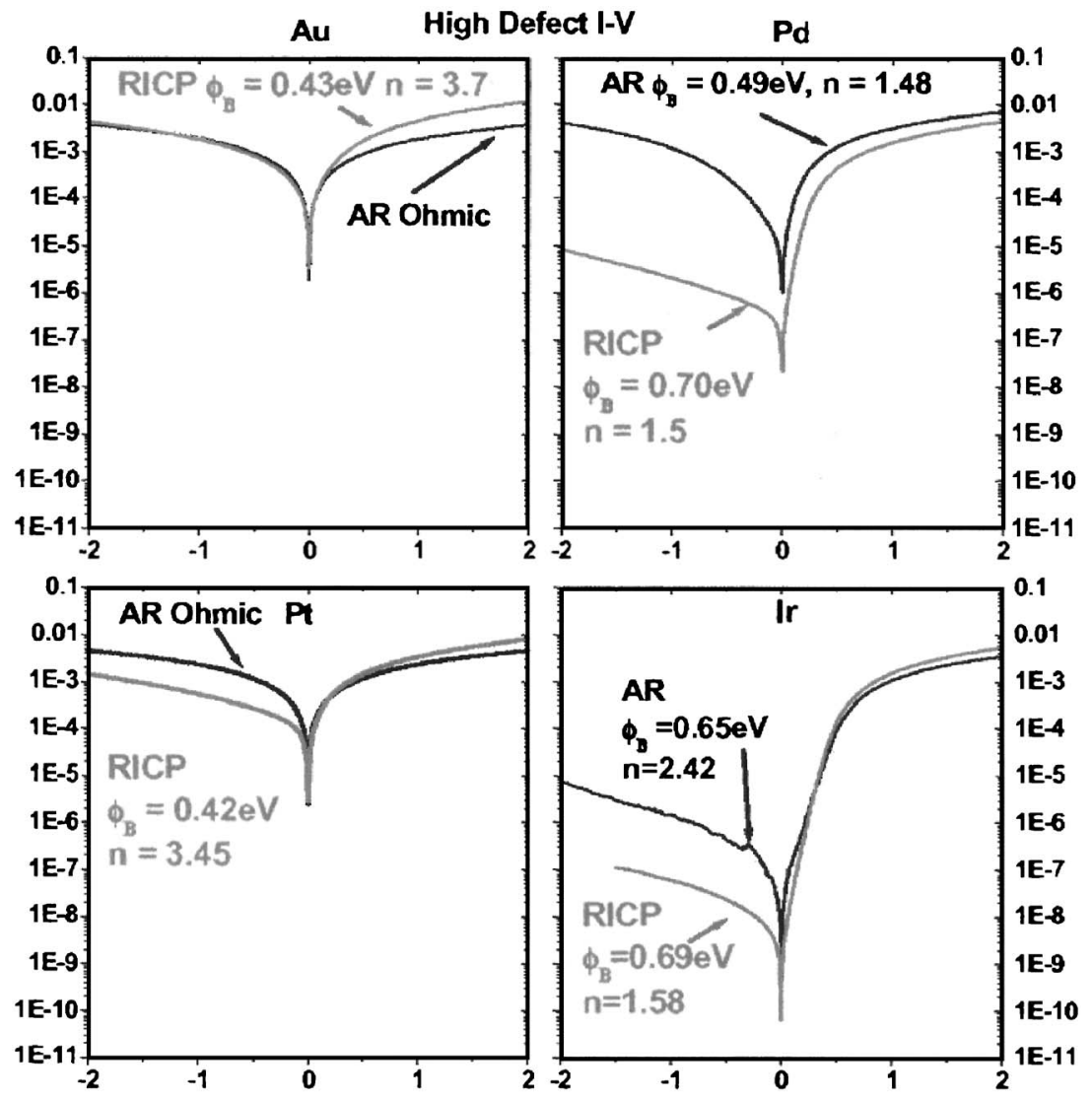

FIG. 6. $\log I-V$ characteristics for Au, $\mathrm{Pd}, \mathrm{Pt}$, and Ir diodes on high defect $\mathrm{AR}$ vs ROP-treated $\mathrm{ZnO}(000 \overline{1})$. For each metal, the ROP treatment increases $\Phi_{B}$ and lowers both reverse leakage current and $n$ values. 
TABLE I. Low defect ZnO Schottky barrier heights and ideality factors for $\mathrm{Au}$, Ir, and Pt before and after $1 \mathrm{~h} \mathrm{O}_{2}$ plasma treatment. In general, ROP treatment raises barriers and lowers ideality factors. For a given treatment, the low defect $\mathrm{ZnO}$ typically exhibits higher $\Phi_{B}$ and lower $n$ values.

\begin{tabular}{ccccc}
\hline \hline Metal & Process & Defect level & Schottky barrier & Ideality \\
\hline $\mathrm{Au}$ & $1 \mathrm{~h} \mathrm{He}-\mathrm{O}_{2}$ ROP & High & 0.43 & 3.57 \\
$\mathrm{Au}$ & $\mathrm{As} \mathrm{received}$ & Low & 0.46 & 1.56 \\
$\mathrm{Au}$ & $1 \mathrm{~h} \mathrm{He}-\mathrm{O}_{2}$ ROP & Low & 0.48 & 1.30 \\
$\mathrm{Ir}$ & $1 \mathrm{~h} \mathrm{He}-\mathrm{O}_{2}$ ROP & High & 0.65 & 2.62 \\
$\mathrm{Ir}$ & $\mathrm{As} \mathrm{received}$ & Low & 0.54 & 1.66 \\
$\mathrm{Ir}$ & $1 \mathrm{~h} \mathrm{He}-\mathrm{O}_{2}$ ROP & Low & 0.64 & 1.36 \\
$\mathrm{Pt}$ & $1 \mathrm{~h} \mathrm{He}-\mathrm{O}_{2}$ ROP & High & 0.42 & 3.45 \\
$\mathrm{Pt}$ & $\mathrm{As} \mathrm{received}$ & Low & Ohmic & NA \\
$\mathrm{Pt}$ & $1 \mathrm{~h} \mathrm{He}-\mathrm{O}_{2}$ ROP & Low & 0.39 & 1.00 \\
\hline \hline
\end{tabular}

and their depth distribution in the subsurface regions. $C$ - $V$ measurements for $\mathrm{Ir}-\mathrm{ZnO}(000 \overline{1})$ diodes with $\Phi_{B}=0.69 \mathrm{eV}$ show carrier density increasing from $3 \times 10^{16} \mathrm{~cm}^{-3}$ at $200 \mathrm{~nm}$ depth to $10^{17} \mathrm{~cm}^{-3}$ at $90 \mathrm{~nm}$ below the metal interface. This increase is mirrored by a similar three times increase in $2.5 \mathrm{eV}$ DL emission observed with DRCLS over the range from 200 to $\sim 50 \mathrm{~nm}$. DLTS measurements of this interface show a deep level with activation energy corresponding to a level $0.53 \mathrm{eV}$ below the conduction band $E_{C}$ with a density of $3.35 \times 10^{14} \mathrm{~cm}^{-3}$. DLOS spectra reveal multiple deep levels across the $\mathrm{ZnO}$ band gap at $E_{C}-1.88$, 2.88 , and $3.14 \mathrm{eV}$ as well as $E_{V}+2.7 \mathrm{eV}$.

In addition to the deep level defects resident in the bulk $\mathrm{ZnO}$, similar defects are observed to form at the metal- $\mathrm{ZnO}$ interface following metal deposition. Such metal-induced states depend on the particular metal. Figure 7 illustrates DRCLS spectra for $\mathrm{Au}$ and $\mathrm{Al}$ diodes deposited on the same low defect $\mathrm{ZnO}(000 \overline{1})$ surface. In the Au case relative to the bare surface nearby (a), Au induces relatively little change in the already low midgap defect emission at $2.5 \mathrm{eV}$ and slight increases at $3 \mathrm{eV}$ and the NBE region. In contrast, $\mathrm{Al}$ in- duces an order of magnitude increase in the $2.5 \mathrm{eV}$ emission as well as small NBE changes relative to the bare surface nearby. Thus Al produces metal-induced deep level defects at its $\mathrm{ZnO}$ interface. Furthermore, the contrast between (a) and (b) shows that this metal-induced defect creation depends on the specific metal.

\section{DISCUSSION}

The results indicate that deep level defects are a common feature of $\mathrm{ZnO}$ single crystals and that they can have a major impact on Schottky barrier properties. The wide variation in deep level defect emission reflected in, e.g., Fig. 2, and the strong correlation of such defects with SB properties in Figs. 4 and 5 underscore the importance of determining the quality of $\mathrm{ZnO}$ used for electrical or optical studies as well as for device structures.

ROP treatment dramatically improves SB characteristics. Figure 3 shows that the conversion from Ohmic to Schottkylike $I-V$ characteristics takes place for exposure times longer than needed to clean the surface, thereby establishing the importance of subsurface defects and impurities such as $\mathrm{H}$. Figure 4 demonstrates the effect of different concentrations of such DL concentrations in the near surface region on electrical properties. The low defect $\mathrm{ZnO}$ has two orders of magnitude lower DL emission versus the high defect $\mathrm{ZnO}$ shown in Fig. 4(a). The corresponding $I-V$ curve in Fig. 4(b) shows a reverse current decrease of 100 times and corresponds to an increase in barrier height from 0.43 to $0.6 \mathrm{eV}$.

$C$ - $V$ measurements confirm our DRCLS results showing an increase in defects and carrier density toward the free $\mathrm{ZnO}$ surface for specific interfaces. Such defects within $\mathrm{ZnO}$ crystals may arise due to nonstoichiometric growth conditions, thermal processing, impurities, or mechanical damage, e.g., surface polishing. Such results provide evidence for either defect diffusion toward the surface or defect creation at the metal interface. Defect creation via chemical bonding between metals and $\mathrm{Zn}$ or $\mathrm{O}$ atoms is readily understood.
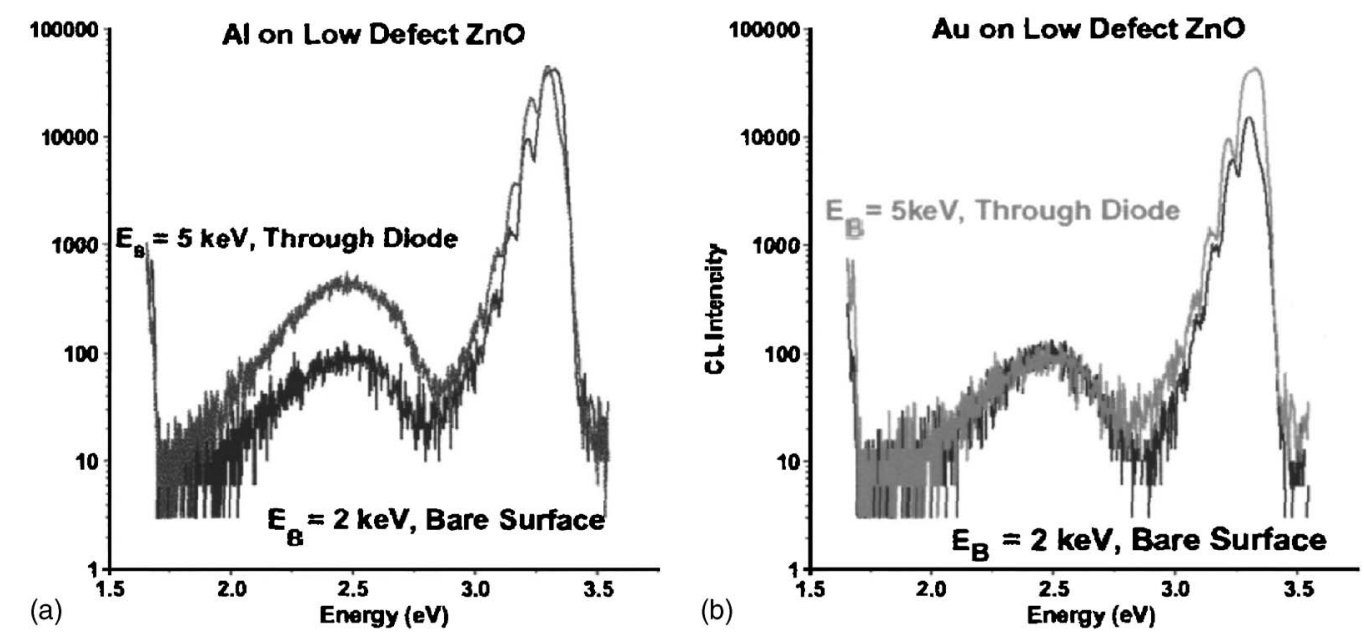

FIG. 7. DRCL spectra compared for (a) Al vs (b) Au on low defect $\mathrm{ZnO}$ after $1 \mathrm{~h}$ ROP treatment. The bare surface defect emission at 2.5 eV is nearly 1000 times lower than NBE peak intensity. Au deposition induces only relatively small changes, particularly around 3 eV and the NBE region. Al deposition on the same surface induces a 10 times increase in $2.5 \mathrm{eV}$ deep level emission. 
However, defect diffusion can also occur-driven, for example, electrically in the case of charged defects. Calculations suggest that vacancy and interstitial diffusion in $\mathrm{ZnO}$ can occur at relatively low temperatures. ${ }^{19}$ For high deep level concentrations, hopping transport can occur that enables tunneling through barriers, increased reverse currents, and effective SB reductions. For shallower levels, such defects can alter the carrier concentration within the near surface depletion region. Thus new donors can increase $n$-type doping near the surface (as observed in our $\mathrm{Ir}-\mathrm{ZnO} C-V$ measurements), thereby narrowing the depletion width and increasing tunneling currents. Temperature-dependent electrical measurements are underway to establish the relative contributions of these mechanisms.

Figure 5(a) reveals that the spectra for the unprocessed $\mathrm{ZnO}$ exhibit the same DL emission for each metal. ROP processed surfaces, however, indicate significantly larger DL variations. The spectra in Fig. 5(b) show the emergence of a shoulder at $2.5 \mathrm{eV}$ for some metals, while others resemble those of the unprocessed surface. This suggests that the contamination layer observed with XPS (Ref. 20) on the unprocessed surface retards any metal-ZnO interaction. Once the ROP treatment removes this layer, metals can react with the surface and generate changes in defect concentration. Since the chemical bonding between different metals with $\mathrm{Zn}$ and $O$ varies considerably, substantial differences are not surprising if specific metal- $\mathrm{ZnO}$ bonding generates particular localized states. Note that metals such as $\mathrm{Au}$ and $\mathrm{Pd}$ that do not bond strongly with $\mathrm{O}$ can also interact with $\mathrm{ZnO}$ since they can induce eutectics such as Au with $\mathrm{Zn}$.

Figure 6 illustrates the improvement in $I-V$ characteristics and SB height with ROP treatment for $\mathrm{Au}, \mathrm{Pd}, \mathrm{Pt}$, and Ir on high defect $\mathrm{ZnO}$. For Pd, Pt, and Ir, large decreases in reverse current are evident after plasma processing. In terms of optimal ZnO SB metallizations. Table I shows that $1 \mathrm{~h}$ ROP treatments on low defect $\mathrm{ZnO}$ produce higher $\mathrm{SBs}$ and lower ideality factors for $\mathrm{Au}$, Ir, and Pt. Likewise, Table I also shows that plasma processing, in general, produces higher barriers, lower reverse currents, and lower idealities for low defect versus high defect $\mathrm{ZnO}$. In addition to the data presented in Table I, we found the following: (1) All metals on AR high defect $\mathrm{ZnO}$ with the exception of Ir and Pd displayed Ohmic characteristics. (2) Ir contacts seemed to have the largest SBs of $0.65 \mathrm{eV}$ for AR high defect $\mathrm{ZnO}$ and 0.69 for its ROP-treated counterpart. The ROP step changed $n$ from 2.42 to 1.58 and decreased the reverse current by two orders of magnitude. Pt diode rectification was poor, with a large $n$ indicating that the contact is dominated by nonthermionic transport. Pd seemed to have the lowest $n$ before and after ROP. Its SB height increased from 0.49 to $0.7 \mathrm{eV}$ with an $n$ relatively unchanged from 1.48 to 1.50 .

Palladium and iridium typically are the best choice metals for high defect material with barrier heights of 0.69 and 0.7 with low idealities. However, SEM images of Ir contacts show that Ir does not adhere well to $\mathrm{ZnO}$ surfaces. The stability of the Ir contact degrades as the metal "peels" away from the semiconductor with time or temperature. ${ }^{18}$
Figure 7 demonstrates that metals do, in fact, induce defects at $\mathrm{ZnO}$ interfaces. Here, the low defect $\mathrm{ZnO}$ permits clear identification of defect changes due to metal interaction that would otherwise be masked by the much higher densities of other crystals. For this low defect, vapor phase transport-grown $\mathrm{ZnO}, \mathrm{Al}$ induces nearly an order of magnitude increase in the $2.5 \mathrm{eV} \mathrm{DL}$ emission within the $25 \mathrm{~nm}$ depth below the metal junction, whereas $\mathrm{Au}$ induces little change. These data clearly show that such metal-induced states depend on the specific metal-ZnO interaction. Indeed, this $2.5 \mathrm{eV}$ emission can be attributed to oxygen vacancies and may arise from $\mathrm{Al}$ reaction that extracts $\mathrm{O}$ from the $\mathrm{ZnO}$ lattice near the interface. Interactions observed at higher temperatures for all these metals suggest chemical trends consistent with this picture. ${ }^{18}$

\section{CONCLUSIONS}

The results presented here demonstrate that high defect $\mathrm{ZnO}$ creates poor rectifying contacts. Defects both at the surface and extending into the bulk affect electrical measurements substantially. Metals induce defects at $\mathrm{ZnO}$ surfaces. These metal-induced states depend on the specific metal interaction. Overall, clean, ordered surfaces of low defect $\mathrm{ZnO}$ produce the highest Schottky barriers and lowest ideality factors for rectifying contacts. Hence, one must consider the role of subsurface bulk defects and metal reactions when fabricating $\mathrm{ZnO}$ electronic devices.

\section{ACKNOWLEDGMENTS}

The authors gratefully acknowledge support for this work by National Science Foundation Grant No. DMR-0513968 (Verne Hess). The authors also thank Michael Callahan (Hanscomb Air Force Base) for representative $\mathrm{ZnO}$ crystals from vendors worldwide.

Presented at the AVS 53rd International Symposium and Exhibition, San Francisco, CA, 12-17 November 2006.

${ }^{1}$ U. Ozgur, Y. I. Alivov, C. Liu, A. Teke, M. A. Reshchikov, S. Dogan, V. Avrutin, S. J. Cho, and H. Morkoc, J. Appl. Phys. 98, 041301 (2005).

${ }^{2}$ S. J. Pearton, D. P. Norton, Y. W. Heo, L. C. Tien, M. P. Ivill, Y. Li, B. S. Kang, F. Ren, J. Kelly, and A. F. Hebard, J. Electron. Mater. 35, 862 (2006).

${ }^{3}$ S. J. Pearton, D. P. Norton, K. Ip, Y. W. Heo, and T. Steiner, Prog. Mater. Sci. 50, 312 (2005).

${ }^{4}$ D. C. Look, J. Electron. Mater. 35, 1299 (2006).

${ }^{5}$ H. von Wenckstern, G. Biehne, and R. A. Rahman, Appl. Phys. Lett. 88, 092102 (2006).

${ }^{6}$ M. W. Allen, M. M. Alkaisi, and S. M. Durbin, Appl. Phys. Lett. 89, 103520 (2006).

${ }^{7}$ F. D. Auret, J. M. Nel, M. Hayes, L. Wu, W. Wesch, and E. Wendler, Superlattices Microstruct. 39, 17 (2006).

${ }^{8}$ T. E. Murphy, J. O. Blaszczak, K. Moazzami, W. E. Bowen, and J. D. Phillips, J. Electron. Mater. 34, 699 (2005).

${ }^{9}$ T. Nagata, P. Ahmet, Y. Z. Yoo, K. Yamada, K. Tsutsui, Y. Wada, and T. Chikyow, Appl. Surf. Sci. 252, 2503 (2006).

${ }^{10}$ K. Ip, G. T. Thaler, H. S. Yang, S. Y. Han, Y. J. Li, D. P. Norton, S. J. Pearton, and S. W. Jang, and F. Ren, J. Cryst. Growth 287, 149 (2006).

${ }^{11}$ B. J. Coppa, C. C. Fulton, S. M. Kiesel, R. F. Davis, C. Pandarinath, J. E. Burnette, R. J. Nemanich, and D. J. Smith, J. Appl. Phys. 97, 103517 (2005).

${ }^{12}$ Y. M. Strzhemechny, H. L. Mosbacker, D. C. Look, D. C. Reynolds, C. 
W. Litton, N. Y. Garces, N. C. Giles, L. E. Halliburton, S. Niki, and L. J. Brillson, Appl. Phys. Lett. 84, 2545 (2004).

${ }^{13}$ Y. M. Strzhemechny, J. Nemergut, P. E. Smith, J. Bae, D. C. Look, and L. J. Brillson, J. Appl. Phys. 94, 4256 (2003).

${ }^{14}$ H. L. Mosbacker, Y. M. Strzhemechny, B. D. White, P. E. Smith, D. C. Look, D. C. Reynolds, C. W. Litton, and L. J. Brillson, Appl. Phys. Lett. 87, 012102 (2005).

${ }^{15}$ B. J. Coppa, C. C. Fulton, P. J. Hartlieb, R. F. Davis, B. J. Rodriguez, B.
J. Shields, and R. J. Nemanich, J. Appl. Phys. 95, 5856 (2004).

${ }^{16}$ A. Hierro, D. Kwon, S. A. Ringel, S. Rubini, E. Pelucchi, and A. Franciosi, J. Appl. Phys. 87, 730 (2000).

${ }^{17}$ D. Drouin, P. Hovington, and R. Gauvin, Scanning 19, 20 (1997).

${ }^{18}$ H. L. Mosbacker, M. Hetzer, and L. J. Brillson (unpublished).

${ }^{19}$ A. Janotti and C. G. Van de Walle, J. Cryst. Growth 287, 58 (2006).

${ }^{20}$ D. C. Look, H. L. Mosbacker, Y. M. Strzhemechny, and L. J. Brillson, Superlattices Microstruct. 38, 406 (2005). 